\title{
OS IMPACTOS DA COVID-19 NA EDUCAÇÃO POR MEIO DO ENSINO REMOTO
}

\author{
THE IMPACTS OF COVID-19 ON EDUCATION THROUGH REMOTE TEACHING
}

\begin{abstract}
Raul Sousa Andreza*a,b, Edislayne Jiselly de Souza Alves ${ }^{b}$, Lidiane Hilário Martins ${ }^{b}$ Renata Hilário Silvab, Shirley Daiane Alves da Silvab, Thalia Lima Nogueirab, Amanda Raquel Novaes Gomes ${ }^{b}$, Janete Clair da Silva Santos ${ }^{b}$
\end{abstract}

Faculdade de Ciências Médicas Aggeu Magalhães, FAMAa Faculdade de Ciências Exatas e Humanas do Sertão Central do São Francico, FACESFb *E-mail: raulsousaandreza@gmail.com

\section{RESUMO}

As ações executadas em função do enfrentamento à pandemia causada pela COVID - 19, apesar de necessárias, conduziram a uma série de consequências em distintos setores principalmente na educação através do distanciamento social, exigindo a implementação do ensino intermediado por tecnologias que por apresentar limitações e falhas ainda se manifesta como um dilema a sociedade acadêmica. O estudo analisou de que forma a educação está sendo afetada perante atual situação do novo Coronavírus e os efeitos no processo de ensino e de aprendizagem por meio de atividades remotas. Pesquisa de caráter descritivo, com abordagem quali-quantitativa, mediante aplicação de um questionário eletrônico por meio da plataforma Google Forms. A amostra foi constituída por 185 acadêmicos das mais diferentes regiões do Brasil, 45,9\% destes apontaram que estão sendo visivelmente impactados pela pandemia e 40,5\% revelaram que por meio do novo método online estão apresentando um desempenho ruim. As maiores dificuldades referiram-se a problemas em nível pessoal e emocional, acesso à internet e a falta de habilidades no uso de tecnologias. Diante dos resultados, $56,4 \%$ dos discentes se posicionaram contra o retorno das atividades de ensino remoto na graduação. Os dados mostraram que apesar da didática virtual representar a melhor alternativa às circunstâncias atuais, a maneira com que a mesma tem se colocado em prática não tem gerado os benefícios esperados, principalmente pelos obstáculos técnicos e estruturais encontrados e bem como as dificuldades individuais de cada estudante que não podem ser resolvidas com uma simples solução empregada de forma generalizada.

Palavras-chave: Coronavírus; Ensino virtual; Discentes.

\section{ABSTRACT}

The actions taken in response to the pandemic caused by COVID - 19, despite being necessary, have led to a series of consequences in different sectors mainly in education through social distancing, requiring the implementation of teaching mediated by technologies that, due to their limitations and flaws still presents itself as a dilemma in academic society. The study analyzed how education is being affected by the current situation of the new Coronavirus and the impacts on the teaching and learning process by remote activities. Descriptive research, with qualiquantitative approach, through the application of an electronic questionnaire using the Google Forms platform. The sample consisted of 185 academics from the most different regions of Brazil, $45.9 \%$ of them pointed out that they are being visibly impacted by the pandemic and $40.5 \%$ revealed that by the new online method they are performing badly. The greatest difficulties were related to problems on a personal and emotional level, access to the internet, and the lack of skills in the use of technologies. Given the results, $56.4 \%$ of the students positioned themselves against the return of the remote teaching activities in graduation. The data showed that although virtual didactics represents the best alternative to current circumstances, the way it has been put into practice has not generated the expected benefits, mainly due to the technical and structural obstacles encountered as well as the individual difficulties of each student that cannot be solved with a simple solution employed in a generalized way.

Keyword: Coronavirus; Virtual teaching; Students. 


\section{INTRODUÇÃO}

Os relatos históricos de pandemias acometem a humanidade há mais de dois mil anos e apesar de seus diferentes contextos históricos bem como diferenças sociais, temporais, geográficas e econômicas, possuem roteiros semelhantes. Destacando-se a gripe espanhola que em consequência do seu quadro clínico e agente etiológico viral se demonstra bastante similar à nova pandemia causada pela COVID - 19, provocando significativas mudanças sociais e comportamentais na população (SANAR, 2020).

Com ascendência na China, especificamente na cidade de Wuhan, o vírus cientificamente identificado como SARS-COV-2 por apresentar um elevado grau de disseminação em 11 de março de 2020, a Organização Mundial de Saúde (OMS) declarou estado pandêmico, alertando todo os países à presença do vírus que em um curto período de tempo atingiu todos os 6 continentes do planeta (OLIVEIRA e MORAIS, 2020).

Frente a este cenário, os impactos gerados pela COVID-19 refletem não somente na saúde como um problema epidemiológico, mas também em diversos outros setores como na economia e educação, e isto consequentemente exigiu das autoridades a implementação de estratégias que visassem minimizar a rápida disseminação do vírus, e uma das mais eficazes e que se encontra atualmente em funcionamento é o distanciamento social. Contudo, tal medida resultou no fechamento de unidades escolares (colégios, faculdades, universidades) e outras instituições, demandando assim formas alternativas a continuidade no processo de ensino e de aprendizagem (SENHORAS, 2020).

A principal delas refere-se à adoção do ensino remoto, que consiste em um modelo educacional online, ou seja, mediado por vias tecnológicas e amplamente utilizado como alternativa emergencial em situações de crise, assim regulamentado pela portaria $\mathrm{N}^{\circ} 343$ do Ministério da Educação ao qual dispõe acerca da substituição das aulas presenciais por aulas em meios digitais enquanto durar a pandemia do Novo Coronavírus (BRASIL, 2020). Atuando assim como politica pública a fim de reduzir danos e acessível a professores e alunos por meio das Tecnologias de Informação e Comunicação (TICs), mas que se aplica de forma temporária, sendo esta uma das principais características que o diferencia do ensino a distância (EAD). Apesar de apresentar diversas limitações e serem inúmeros os desafios enfrentados, a educação remota se mostra como principio relevante no que diz respeito ao contínuo vinculo entre discentes e docentes.

O estudo analisou de que forma a educação está sendo afetada perante atual situação do novo Coronavírus e os efeitos no processo de ensino e de aprendizagem por meio de atividades remotas.

\section{METODOLOGIA}

Trata-se de um estudo descritivo, com abordagem quali-quantitativa. Quanto ao desenvolvimento no tempo é um estudo transversal (FONTELLES et al., 2009). A amostra do estudo foi constituída por 185 acadêmicos das mais diferentes regiões do Brasil e a coleta de dados se baseou na aplicação de um questionário por meio da ferramenta Google Forms, que ficou disponível por um período de 6 dias. Todos os participantes responderam a um quesito específico do formulário digital para permitir a utilização de suas respostas, utilizado assim como Termo de Consentimento Livre e Esclarecido (TCLE), cumprindo o disposto na Resolução $n^{\circ}$ 466/12 do Conselho Nacional de Saúde (BRASIL, 2012), baseando-se também na Resolução $n^{\circ} 510 / 16$ que respalda os projetos com formulário eletrônico (BRASIL, 2016).

\section{RESULTADOS E DISCUSSÕES}

Durante o período em que o questionário ficou disponível foram alcançados digitalmente 185 graduandos, sendo que todos consentiram a utilização dos dados e responder corretamente todos os quesitos. Com uma amostra de estudo predominantemente feminina $(73,5 \%)$ a pesquisa abrangeu em sua maioria, acadêmicos entre 19 e 30 anos (tabela 1). Resultados semelhantes foram encontrados por Augusto (2020) que analisou a adesão do ensino remoto entre os discentes e docentes, apresentando um público alvo prevalecente entre 18 e 26 $\operatorname{anos}(74,8 \%)$.

Dentre os estados aos quais os participantes residem, o mais recorrente foi Pernambuco (79\%) seguido pelos estados da Paraíba (6\%), Bahia (5\%), Ceará (3\%), Amazonas (2\%), Piauí (2\%), Alagoas (1\%), Mato Grosso do Sul (1\%), Distrito Federal (1\%), São Paulo (1\%) e Rio Grande do Norte (1\%).

Em relação às áreas de graduação tem-se: Biológicas/Saúde $(67,6 \%)$, Humanas $(14,1 \%)$, Exatas $(12,4 \%)$ e outras $(5,9 \%)$, tendo em vista a prevalência de alunos do curso da área de Biológicas/Saúde, a aplicação da modalidade virtual para tal setor seria parcialmente inadequada por apresentar uma extensiva e importante carga horária no campo prático, no qual o educando adquire conhecimentos que são impossíveis de se obter em sala de aula e que serão amplamente utilizados em sua vida profissional, ou seja, ainda como medida emergencial durante o período pandêmico persiste essa lacuna no desenvolvimento acadêmico (COSTA et al., 2020). 
Tabela 1. Distribuição dos graduandos em relação ao gênero, idade e área de graduação, Julho 2020.

\begin{tabular}{llcc}
\hline & & n & $\%$ \\
Gênero & Feminino & 136 & 73,5 \\
Total & Masculino & 49 & 26,5 \\
Faixa-etária & & $\mathbf{1 8 5}$ & $\mathbf{1 0 0}$ \\
& 18 anos ou menos & 16 & 8,6 \\
& Entre 19 e 30 anos & 144 & 77,9 \\
Total & Entre 31 e 45 anos & 25 & 13,5 \\
Área de graduação & Acima de 46 anos & 0 & 0 \\
& & $\mathbf{1 8 5}$ & $\mathbf{1 0 0}$ \\
& Biológicas $\backslash$ Saúde & 125 & 67,6 \\
& Humanas & 26 & 14,1 \\
Total & Exatas & 23 & 12,4 \\
& Outras & 11 & 5,9 \\
\hline
\end{tabular}

Fonte: Dados da pesquisa

Considerando o total de respostas avaliadas, $80 \%$ dos acadêmicos confirmaram o funcionamento do ensino remoto em suas respectivas instituições educacionais, apenas 20\% negaram utilização de tal método, comprovando assim a ampla aderência no uso de atividades remotas em diversos estados brasileiros mediante atual situação de pandemia ao qual estamos situados, efetivado com o intuito de representar uma politica de redução de danos temporária ao processo de ensino e de aprendizagem. Contudo, $60 \%$ das universidades públicas não aderiram à recomendação do Ministério da Educação (MEC) como publicado por Palhares (2020), justificando ser incapaz de ofertar atividades com a mesma qualidade que o ensino presencial e bem como impossibilidade de garantir o alcance do material pedagógico a todos os educandos.

Tendo-se por base os dados gerados pelos acadêmicos que confirmaram a utilização do método de ensino remoto em suas respectivas instituições educacionais, $45,9 \%$ responderam que a pandemia está afetando seus estudos de forma muito negativa e que não conseguem estudar. Seguido por $19,6 \%$ que alegaram indiferença, não tendo a atual crise alterado em nada no aprendizado, 17,6\% manifestaram indisponibilidade de tempo qualificando como ruim e por fim, $16,9 \%$ contestaram ter até mais tempo disponível para estudar (tabela 2).

Uma pesquisa divulgada pela Associação Brasileira de Mantenedoras de Ensino Superior (ABMES) revelou resultados opostos em que a percepção dos estudantes é vista de forma diferente, $52 \%$ avaliaram que a pandemia teve pouco impacto, enquanto que, $30 \%$ descreveram um significativo efeito sobre sua rotina (ABMES, 2020). Além disso, mundialmente mais de $91 \%$ da comunidade estudantil foi de alguma forma afetada em decorrência do fechamento das instituições, transformando-se assim em um período perturbador para muitos, e por este motivo a Organização das Nações Unidas para a Educação, a Ciência e a Cultura (UNESCO) tem apoiado diversos países a enfrentar as crises internas, principalmente no setor educacional dando suporte à continuidade do ensino por meio de plataformas digitais (PRAGHOLAPATI, 2020). Com relação às consequências pós-pandêmicas que o país enfrentará a pesquisa desenvolvida por Freitas (2020) mostrou que $52 \%$ dos entrevistados estão pessimistas, apenas $16 \%$ otimistas, enquanto que $25 \%$ e $8 \%$, respectivamente, se declaram neutros e não souberam responder.

Tabela 2. Posicionamento dos discentes sobre o quão impactante está sendo a pandemia nos estudos

\begin{tabular}{lcc}
\hline \multicolumn{1}{c}{ Classificação } & \multicolumn{2}{c}{ Frequência } \\
De forma muito negativa, não consigo estudar & 68 & $\%$ \\
Indiferente, não alterou em nada meu ritmo de estudo & 29 & 45,9 \\
Ruim, não tenho tempo disponível para estudar & 26 & 17,6 \\
Tenho mais tempo disponível para estudar & 25 & 16,9 \\
Total & 148 & 100 \\
\hline
\end{tabular}


Em relação ao ponto de vista dos discentes sobre seu rendimento acadêmico perante a didática virtual, apenas 3,4\% dos participantes registraram como ótimo seu desempenho, já 40,5\%, 38,5\% e 17,6\% apontaram respectivamente como ruim, regular e bom o aproveitamento acadêmico durante esse semestre. Desfecho diferente foi citado ainda na pesquisa da Associação Brasileira de Mantenedoras de Ensino Superior, em que 59\% aprovaram e destacaram como positiva a experiência, 18\% disseram ser indiferentes em relação ao novo formato e $23 \%$ julgaram como negativa (ABMES, 2020).

Diversas adversidades foram relatadas e dentre elas, destacaram-se as de ordem pessoal e emocional com um percentual de 35,8\%. O estudo proposto por Maia e Dias (2020) apontou os impactos da COVID-19 sobre a saúde mental de universitários em comparação com anos anteriores, demonstrando a gradual incidência de ansiedade, depressão e estresse, fatores estes diretamente relacionados ao que foi exposto pelos participantes do presente estudo, aliado aos efeitos perturbadores do isolamento social.

A segunda maior complicação citada pelos educandos foi a grande quantidade de atividades exigidas pelos professores $(31,1 \%)$, fato também apontado por Hortegal (2020) no qual 33\% dos estudantes se referiram ao excesso de material didático. Diante disso, Torres et al (2020) retrata esse árduo processo adaptativo a essa nova realidade, principalmente no que diz respeito ao desenvolvimento de dinâmicas e atividades que se engajem nesse modelo de ensino, sem promover prejuízos aos estudantes, ou seja, os docentes também estão diante de um enorme desafio pela promoção e disseminação de conhecimento.

Seguido por um dos grandes obstáculos enfrentado não só por estudantes, mas toda a sociedade civil, que é o acesso a internet (9,5\%). Reforçando assim as desigualdades sociais uma vez que nem toda a comunidade acadêmica tem pleno acesso a esse serviço, como demonstra dados de Augusto (2020), em que dos 3,3 mil graduandos que responderam ao questionário pelo menos 17,2\% relataram tal impasse. Apesar de ser uma ferramenta fundamental para execução desse método de ensino com aproximação virtual, uma a cada quatro pessoas no Brasil não tem acesso à internet, como divulgado pela Pesquisa Nacional por Amostra de Domicílios Contínua - Tecnologia da Informação e Comunicação (Pnad Contínua TIC) 2018 e exposta pelo Instituto Brasileiro de Geografia e Estatística (IBGE), em números totais, isso representa cerca de 46 milhões de brasileiros ( $25 \%$ da população) que não acessam a rede (TOKARNIA, 2020). Além de que para 5,7\% o equipamento necessário para a navegação é consideravelmente caro se tornando assim indisponível, fato também evidenciado na pesquisa, no qual 10,8\% deles indicaram ter problemas de disponibilidade $\mathrm{e}$ funcionamento de equipamentos (tabela 3), visando tal perspectiva que governos internacionais estão estabelecendo medidas para ampliar o alcance técnico aos equipamentos promovendo equidade educacional (ARRUDA, 2020).

Tabela 3. Distribuição das dificuldades enfrentadas no encerramento do semestre 2020.1 no que diz respeito ao ensino remoto

\section{Principais obstáculos apontados pelos graduandos}

\section{Frequência}

n

Dificuldades em ordem pessoal e emocional

Volume de atividades exigidas pelos docentes

Problemas com a disponibilidade e o funcionamento de equipamentos

Problemas com acesso a internet

Não tive dificuldades

Falta de habilidade no uso de tecnologias de informação e comunicação

$\begin{array}{rl}53 & 35,8 \\ 46 & 31,1 \\ 16 & 10,8 \\ 14 & 9,5 \\ 10 & 6,8 \\ 9 & 6,0\end{array}$

148100

Fonte: Dados da pesquisa

Outros estudos apontam desafios relacionados a essa alternativa emergencial, como a visível diferença no contato e interação entre professor e aluno, ao qual se associa a dificuldade de manter os estudantes concentrados e atentos ao que é transmitido pela tela do aparelho, surgindo assim outro impasse que é a instabilidade da conexão (XIAO e LI, 2020).

Por fim, este estudo também analisou a posição dos discentes com relação ao retorno das atividades de ensino na graduação de forma remota, em que $51,4 \%$ 
destes não apoiam tal medida, 37,8\% responderam que sim, enquanto que $10,8 \%$ afirmaram que essa solução não se aplica a realidade do curso ao qual atendem. Sendo que o estudo realizado por Alvim (2020) revelou que $73 \%$ dos alunos que responderam ao questionário de enquete são favoráveis ao continuo funcionamento dessa modalidade e apenas $25,5 \%$ não estão de acordo com esta deliberação. No estudo elaborado por Hortegal (2020) foi exposto que pelo menos $45,6 \%$ alegaram apoiar a continuidade na utilização de recursos digitais no meio acadêmico, 29,8\% contestaram que não e por último $24,6 \%$ responderam que tal didática não se adequa ao curso.

Gráfico 1. Ponto de vista dos participantes sobre o retorno das atividades de forma remota na graduação

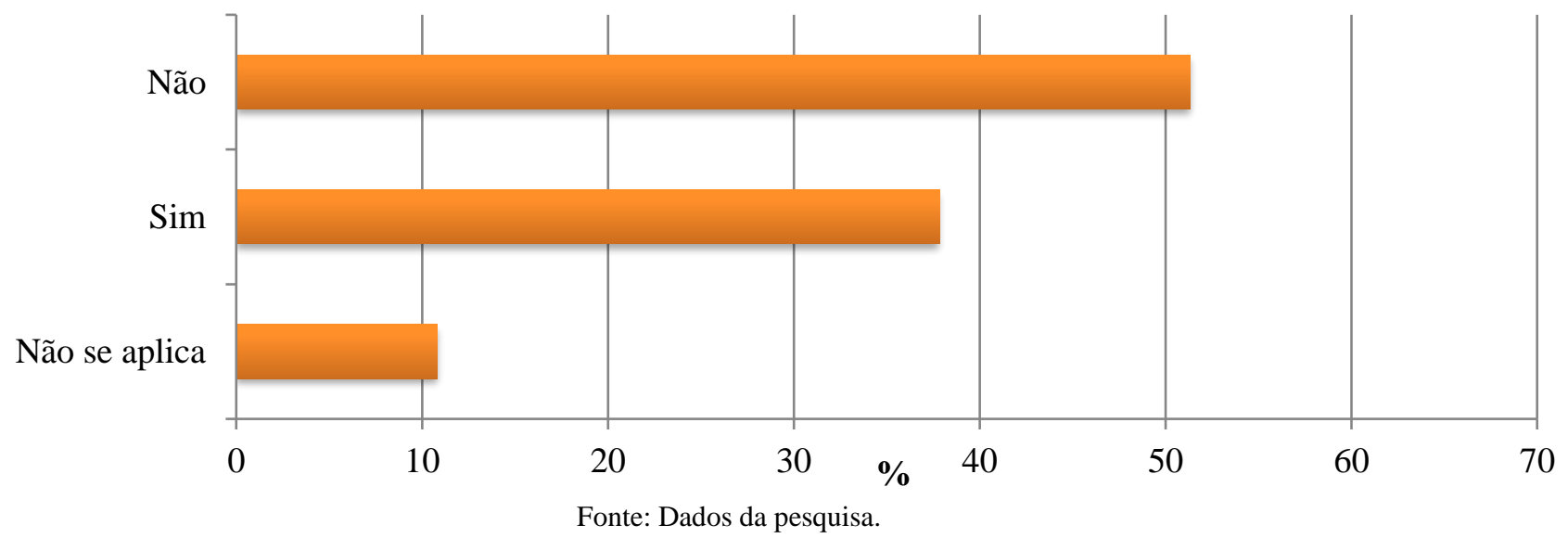

\section{CONCLUSÃO}

Diante do estudo preliminar, fez-se notório os impactos da pandemia causada pela COVID - 19 que impulsionou não só a crise econômica já instaurada no país, como também alavancou as desigualdades sociais e colocou em prova nosso fragilizado sistema de saúde. A educação foi decerto proporcionalmente afetada em que se empregou a aplicação do ensino remoto.

Com base nos resultados obtidos por esta pesquisa confirmou-se que muitas instituições já aderiram a tal proposta, contudo, analisando a classificação dos estudantes que se revelaram insatisfeitos apontando como negativo o seu rendimento, diversos foram os obstáculos encontrados além da inacessibilidade parcial a internet, a falta de habilidades no uso de tecnologias e os problemas pessoais e emocionais.

Em termos de efetividade a proposta de ensino remoto apresenta-se como um plano provisório que poderá manifestar-se positivamente, mas que por apresentar problemas técnicos e estruturais não consegue englobar a todos, sendo necessário colocar em pauta de que forma esse processo está sendo inserido e efetivado no meio acadêmico e quais são as medidas mais adequadas para melhorar a utilização dessa alternativa pedagógica. Além disso, é imprescindível o apoio à continuidade na produção literária que envolve tal temática.

\section{REFERÊNCIAS}

AUGUSTO, A.S. Pesquisa revela principais dificuldades dos alunos do IFFar nas atividades remotas. Instituto Federal Farroupilha, Rio Grande do Sul, 7 de abril de 2020. Disponível https://www.iffarroupilha.edu.br/noticiassau/item/17146-pesquisa-revela-principais-dificuldadesdos-alunos-do-iffar-nas-atividades-remotas-

5e8e8faba9a74. Acesso em: 19 de julho de 2020.

ALVIM, A.E. Pesquisa do MEC com estudantes e professores do ensino superior busca avaliar estudo remoto - respostas até 31/7. Universidade Federal de Lavras, Minas Gerais, 16 de julho de 2020. Disponível em: https://ufla.br/noticias/ensino/13879-pesquisa-domec-com-estudantes-e-professores-do-ensino-superiorbusca-avaliar-estudo-remoto-respostas-ate-31-7. Acesso em: 19 de julho de 2020.

ARRUDA, E.P. EDUCAÇÃO REMOTA EMERGENCIAL: elementos para políticas públicas na educação brasileira em tempos de Covid-19. EmRede, v.7, n.1, 2020. 
BRASIL, Ministério da Educação. Portaria nº 343, de 17 de março de 2020. Disponível em:<http://www.in.gov.br/en/web/dou/-/portaria-n-343de-17-de-marco-de-2020-248564376> Acesso em 8 de julho de 2020.

BRASIL, Resolução $\mathrm{n}^{\circ}$ 466, de 13 de junho de 2012. Dispõe sobre diretrizes e normas regulamentadoras de pesquisas envolvendo seres humanos. Diário Oficial [da] República Federativa do Brasil, Brasília, 14 de junho de 2013. Acesso em: 8 de julho de 2020.

BRASIL, Resolução n ${ }^{\circ}$ 510, de 07 de abril de 2016. Dispõe sobre as normas aplicáveis a pesquisas em Ciências Humanas e Sociais. Diário Oficial [da] República Federativa do Brasil, Brasília, DF, 24 de maio 2016. Acesso em: 8 de julho de 2020.

COSTA et al. Ensino de enfermagem em tempos de covid19: como se reinventar nesse contexto?. Texto \& Contexto Enfermagem, Florianópolis, v. 29, 2020.

FREITAS, S. de. Pesquisa aponta impactos da pandemia sobre a comunidade universitária. Universidade Federal do Espírito Santo, Espírito Santo, 27 de abril de 2020. Disponível em: http://www.ufes.br/conteudo/pesquisaaponta-impactos-da-pandemia-sobre-comunidadeuniversitaria> Acesso em: 19 de julho de 2020.

FONTELLES, et. al. Metodologia da pesquisa científica: Diretrizes para a elaboração de um protocolo de pesquisa. Revista paraense de Medicina, v.23, n.3, 2009.

ABMES. Na pandemia, 22\% das faculdades particulares pausaram atividades e não adotaram o ensino remoto, diz pesquisa. Associação Brasileira de Mantenedoras de Ensino Superior, Distrito Federal, 7 de maio de 2020. Disponível

em:< https://abmes.org.br/noticias/detalhe/3768/na-pandemia22-das-faculdades-particulares-pausaram-atividades-enao-adotaram-o-ensino-remoto-diz-pesquisa> Acesso em 16 de julho de 2020.

HORTEGAL, S. Pesquisa mede adesão de alunos e professores da UFMA ao ensino remoto. Universidade Federal do Maranhão, Maranhão, 19 de maio de 2020. Disponível em: https://portais.ufma.br/PortalUfma/paginas/noticias/notici a.jsf?id=56256> Acesso em 20 de julho de 2020 .

MAIA, B.R.; DIAS, P.C. Ansiedade, depressão e estresse em estudantes universitários: o impacto da COVID-19. Estud. psicol., Campinas, v.37 , 2020.

OLIVEIRA, E.S.; MORAIS, A.C.L.N. de. COVID-19: uma pandemia que alerta à população. InterAm J Med Health, v.3, 2020.

PALHARES, I. $60 \%$ das universidades federais rejeitam ensino a distância durante quarentena. Folha de S. Paulo, São Paulo, 31 de março de 2020. Disponível em:< https://www1.folha.uol.com.br/educacao/2020/03/60universidades-federais-rejeitam-ensino-a-distanciadurante-quarentena.shtml> Acesso em: 9 de julho de 2020.

PRAGHOLAPATI, A. Covid-19 Impact On Students. Department of Nursing, Faculty of Sport Education and Health, Bandung, Indonesia, 2020.

SANAR. Pandemias na História: o que há de semelhante e de novo na Covid-19. Sanar Saúde, 7 de abril de 2020. Disponível em: https://www.sanarmed.com/pandemiasna-historia-comparando-com-a-covid-19. Acesso em: 14 de julho de 2020.

SENHORAS, E. M. Coronavírus e educação: análise dos impactos assimétricos. Boletim de conjuntura (BOCA), Boa Vista, vol.2, n.5, 2020.

TORKANIA, M. Um em cada 4 brasileiros não tem acesso à internet, mostra pesquisa. Agência Brasil, Rio de Janeiro, 29 de abril de 2020. Disponível em: https://agenciabrasil.ebc.com.br/economia/noticia/202004/um-em-cada-quatro-brasileiros-nao-tem-acessointernet. Acesso em: 16 de julho de 2020.

TORRES, et. al. Educação e Saúde: reflexões sobre o contexto universitário em tempos de COVID-19. 2020.

XIAO, C; LI, Y. Analysis on the Influence of Epidemic on Education in China. "Covid-19 and Student Focused Concerns: Threats and Possibilities", Veena Das and Naveeda Khan, eds., American Ethnologist website, 2020. 